\title{
Determining Equi-Biaxial Residual Stress and Mechanical Properties From the Force-Displacement Curves of Conical Microindentation
}

\author{
J. Yan \\ University of Delaware \\ X. Chen \\ Columbia University \\ Anette M. Karlsson \\ Cleveland State University, a.karlsson@csuohio.edu
}

Follow this and additional works at: https://engagedscholarship.csuohio.edu/enme_facpub

Part of the Mechanical Engineering Commons

How does access to this work benefit you? Let us know!

\section{Original Citation}

Yan, J., Chen, X., and Karlsson, A. M., 2007, "Determining Equi-Biaxial Residual Stress and Mechanical Properties from the Force-Displacement Curves of Conical Microindentation," Journal of Engineering Materials and Technology, 129(2) pp. 200-206.

This Article is brought to you for free and open access by the Mechanical Engineering Department at EngagedScholarship@CSU. It has been accepted for inclusion in Mechanical Engineering Faculty Publications by an authorized administrator of EngagedScholarship@CSU. For more information, please contact library.es@csuohio.edu. 


\begin{abstract}
Determining Equi-Biaxial Residual Stress and Mechanical Properties From the

Department of Mechanical Engineering, University of Delaware, Newark, DE 19716-3140

X. Chen
Department of Civil Engineering and Engineering
Mechanics,
Columbia University,
New York, NY 10027-6699

A. M. Karlsson
Department of Mechanical Engineering,
University of Delaware,
Newark, DE 19716-3140
e-mail: karlsson@udel.edu

\section{Force-Displacement Curves of Conical Microindentation}

An alternative, improved method to determine mechanical properties from indentation testing is presented. This method can determine the elastic modulus, yield strength and equi-biaxial residual stress from one simple test. Furthermore, the technique does not require the knowledge of the contact area during indentation, a parameter that is hard to determine for highly elastic material. The evaluation technique is based on finite element analyses, where explicit formulations are established to correlate the parameter groups governing indentation on stressed specimens.
\end{abstract}

Keywords: microindentation, residual stress, finite element analysis

\section{Introduction}

Microindentation has evolved as a popular technique to measure elastic-plastic material properties [1]. In order to extract these properties, the relationship between the indentation force, $P$, and indentation depth, $\delta$, during loading and unloading (Fig. 1) is measured during the experiment. In addition, the projected contact area, i.e., the projected contact area between the indenter and the substrate during maximum load, is a parameter needed when evaluating the indentation test. However, this area is hard to measure. Thus, we will here present an alternative method to evaluate an indentation test, without the need of the contact area at maximum indentation load.

In evaluating the results, the indenter is usually assumed as a rigid cone. Here we will assume that the cone has a half apex angle $\alpha=70.3^{\circ}$ (equivalent to the Berkovich indenter). In the absence of residual stress, the classic indentation theory relates the hardness, $H$, and contact stiffness, $S$, with yield strength, $\sigma_{y}$, and Young's modulus, $E$, for a homogeneous, isotropic bulk material as:

$$
H=P /\left(\pi a^{2}\right)=c \sigma_{y}
$$

and

$$
S=2 \gamma a E /\left(1-\nu^{2}\right)
$$

Here, $S$ is the slope of the initial portion of the elastic unloading curve, $c$ is a constraint factor that increases with $E / \sigma_{y}, \gamma \approx 1.08$ is a correction factor for conical indenter, and $\nu$ is Poisson's ratio of the homogeneous, isotropic specimen [2,3]. Finally, $a$ is the projected contact radius measured at maximum penetration as indicated in Fig. 1. A common approximation used for determining $a$ is the expression for the contact area, $A$, for a geometrically perfect conical indenter [1]:

$$
A=\pi a^{2}=24.5 \delta_{c}^{2}
$$

where contact depth, $\delta_{c}$, can be determined by [1]

\footnotetext{
Corresponding author.

Contributed by the Materials Division of ASME for publication in the JoURNAL of Engineering Materials and TeChnology. Manuscript received April 5, 2006; final manuscript received June 19, 2006. Review conducted by Assimina Pelegri.
}

$$
\delta_{c}=\delta_{\max }-\varepsilon \frac{P_{\max }}{S},
$$

with $\varepsilon=0.75$ for a Berkovich indenter [1] and $\delta_{\max }$ being the maximum indentation depth (Fig. 1). When the indentation depth is sufficiently large, such that the strain gradient effect may be ignored, both hardness and stiffness are independent of the indentation depth.

It is clear from Eqs. (1) and (2) that both hardness and contact stiffness critically depend on the accuracy of the contact radius $a$, which is closely related with the plastic pile-up (or elastic sinkin), $\delta_{p}$, when $\sigma_{y} / E$ is small (or large) (cf. Fig. 1). It is extremely hard to accurately determine the projected contact radius ${ }^{2}$ [4]. Several authors (e.g., $[1,5,6]$ ) have also pointed out that, even though commonly used, the approximation of the contact radius in Eq. (2) may not be suitable for a range of cases.

We have previously showed that the effect of an equi-biaxial residual stress, $\sigma_{\text {res }}$, has a significant influence on the measured values during indentation testing [4]. For example, we showed that with increasing (more tensile) residual stress the plastic zone gets larger, leading to an apparent lower hardness, whereas with decreasing (more compressive) residual stress the pile-up is enhanced and the plastic zone shrinks, leading to apparent increasing hardness. Therefore, both hardness, $H$, and stiffness, $S$, are sensitive to the magnitude and the size of the residual stress. Equations (1) and (2) do not consider residual stresses in the tested material. With residual stresses being a critical parameter governing mechanical reliability, several efforts have been made to extend these basic equations to allow indentation tests to reveal residual stresses in addition to the basic elastic-plastic properties [4,6-14]. Some of these techniques require testing on a stress-free specimen as a reference, whereas others rely on accurate measurement of either the contact area or the plastic pile-up. These additional measurements are not practical-and may not even be possible-in many cases, making instrumented indentation unnecessarily complicated or even impossible to perform.

${ }^{2}$ It is not practical to measure $a$ at maximum load. The elastic recovery upon unloading can be significant for materials with large $\sigma_{y} / E$, and the elastic recovery also increases with increasing residual compression [4]. Therefore, measuring the contact radius after unloading (e.g., through a surface scan) may lead to substantial errors. 


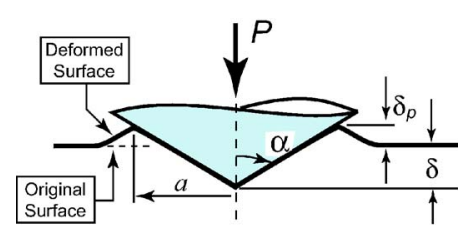

(a)

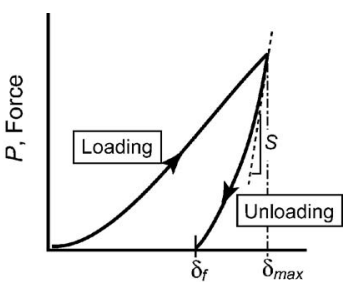

(b) (displacement)

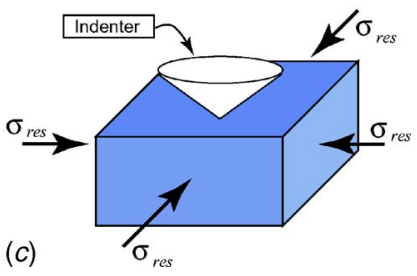

Fig. 1 Schematic of instrumented indentation with a sharp indentation: (a) indentation on a homogeneous, isotropic semiinfinite substrate; (b) typical force-displacement curves obtained from an indentation experiment; and (c) conical indentation on a specimen with equi-biaxial in-plane residual stress

Assuming linear-elastic, perfectly plastic material with a residual stress, there are three unknown parameters that need to be determined: the elastic modulus $E$, the yield strength $\sigma_{y}$, and the residual stress $\sigma_{\text {res }}$. In order to measure all three unknown parameters from one indentation test, one more independent equation is needed in addition to Eqs. ( $1 a)$ and $(1 b)$. This additional equation could be the variation of pile-up with residual stress and elasticplastic properties. However, since the contact radius $a$ is difficult to measure accurately in experiments, it is more desirable to develop new formulations based on the indentation depth $\delta$ instead of $a$. This paper will present an alternative method, eliminating the use of the contact radius, when evaluating the unknown material properties $\left(E, \sigma_{y}, \sigma_{\text {res }}\right)$ from only the force-displacement curve of a conical indentation test.

\section{Model}

This work focuses on microindentation on a linear-elastic, perfectly plastic bulk material with equi-biaxial residual stress. The results are applicable to coating/substrate systems, as long as the substrate effect is small, that is, when the coating is softer than the substrate and the indentation depth is less than $50 \%$ of the coating thickness [15]. The equi-biaxial residual stress in coatings is most commonly generated by thermal expansion mismatch and is critical to system integrity. A schematic of the axisymmetric model used is shown in Fig. 1(c). By ignoring the minor effects from Poisson's ratio and friction [16], dimensional analysis leads to:

$$
\begin{aligned}
\frac{P}{\pi a^{2}} & =\sigma_{y} f\left[\frac{\sigma_{r e s}}{\sigma_{y}}, \frac{\sigma_{y}}{E}\right] \\
\delta_{p} & =\delta g\left[\frac{\sigma_{r e s}}{\sigma_{y}}, \frac{\sigma_{y}}{E}\right] \\
\frac{S}{\delta_{\max }} & =\bar{E} \Psi\left[\frac{\sigma_{r e s}}{\sigma_{y}}, \frac{\sigma_{y}}{E}\right]
\end{aligned}
$$

where $a=\left(\delta_{p}+\delta\right) \tan \alpha$ (Fig. 1) and $\bar{E}=E /\left(1-\nu^{2}\right)$. The normalized indentation work during loading, $W_{l}$, is

$$
\frac{W_{l}}{\delta_{\max }^{3}} \equiv \frac{\int_{0}^{\delta_{\max }} P d \delta}{\delta_{\max }^{3}}=\frac{\pi \tan ^{2} \alpha}{3} \sigma_{y} f\left[\frac{\sigma_{r e s}}{\sigma_{y}}, \frac{\sigma_{y}}{E}\right]\left(1+g\left[\frac{\sigma_{r e s}}{\sigma_{y}}, \frac{\sigma_{y}}{E}\right]\right)^{2}
$$

or

$$
\frac{W_{l}}{\delta_{\max }^{3}}=\sigma_{y} \Pi\left[\frac{\sigma_{r e s}}{\sigma_{y}}, \frac{\sigma_{y}}{E}\right]
$$

This integral contains critical information about the curvature of the loading curve $(P-\delta$-curve, Fig. $1(b))$ and it integrates the important dependence on pile-up, yet without the need to measure the pile-up.

Similarly, the curvature of unloading may be represented by the elastic work, $W_{u}$, recovered during unloading:

$$
\frac{W_{u}}{\delta_{\max }^{3}} \equiv \frac{\int_{\delta_{f}}^{\delta_{\max }} P d \delta}{\delta_{\max }^{3}}=\sigma_{y} \Omega\left[\frac{\sigma_{r e s}}{\sigma_{y}}, \frac{\sigma_{y}}{E}\right]
$$

By varying $\sigma_{r e s} / \sigma_{y}$ and $\sigma_{y} / E$ in a wide range, the dimensionless functions contact stiffness, $\Psi$, work of indentation, $\Pi$, and unload work, $\Omega$, can be determined from extensive finite element analysis. The left sides of (5)-(7) may be readily determined from an indentation experiment and do not require the measurement of either contact radius or pile-up. Finally, by using the three governing equations (5)-(7), the elastic-plastic properties and residual stress may be solved concurrently from reverse analysis. This methodology will be described in the following sections.

\section{Numerical Results}

Finite element calculations were performed using the commercial code ABAQUS [17]. The rigid contact surface option was used to simulate the rigid indenter and the option for finite deformation and strain was employed. A typical mesh for the axisymmetric indentation model comprises more than 5000 eight-node elements with reduced integration and is shown in Fig. 2, for maximum indentation depth. The dimension of the mesh is 4 by $4 \mathrm{~mm}^{2}$. The indentation depth is $25 \mu \mathrm{m}$. In Fig. 2, the boundary conditions are noted, with the symmetric axis on the left side. The bottom is fixed in the $y$-direction and right boundary is fixed in the $x$-direction. Coulomb's friction law is used between the contact surfaces, with the friction coefficient 0.1 (almost frictionless) [18]. Friction between the contact surfaces is a minor factor for indentation $[16,19]$ as long as this value is relatively small. This has been verified by our FEM analyses (not shown for brevity). Extensive studies on the friction effect for conical indentation have also been carried out (e.g., Bucaille et al. [20] and Wang et al. [21], who found similar results). The equi-biaxial in-plane residual stress is applied to the specimen by means of thermal expansion, followed by conical indentation on the free surface. To obtain the numerical values of $\Psi, \Pi$, and $\Omega$, the variables $E / \sigma_{y}$ and $\sigma_{r e s} / \sigma_{y}$ are varied from 10 to 1000 and -1 to 1 , respectively, to cover most combinations of mechanical properties and residual stress encountered in engineering materials.

The finite element results along with the functional forms of $\Psi$, $\Pi$, and $\Omega$ are summarized in Figs. $3(a)-3(c)$, respectively. With increasing Young's modulus, more work (larger force) is needed to indent the specimen to the same depth, which causes the normalized indentation work $\Pi$ to increase with $E / \sigma_{y}$, Fig. 3(b). In addition, residual tension intends to facilitate the penetration by reducing the pile-up, thus decrease the indentation work, $\Pi$, (Fig. $3(b))$. When $E / \sigma_{y}$ is small the material behaves more elastically, allowing for more elastic work, $\Omega$, to be recovered during unloading (Fig. 3(c)). The normalized contact stiffness (Fig. 3(a)) is sensitive to both $E / \sigma_{y}$ and $\sigma_{r e s} / \sigma_{y}$, and increases with the elastic 


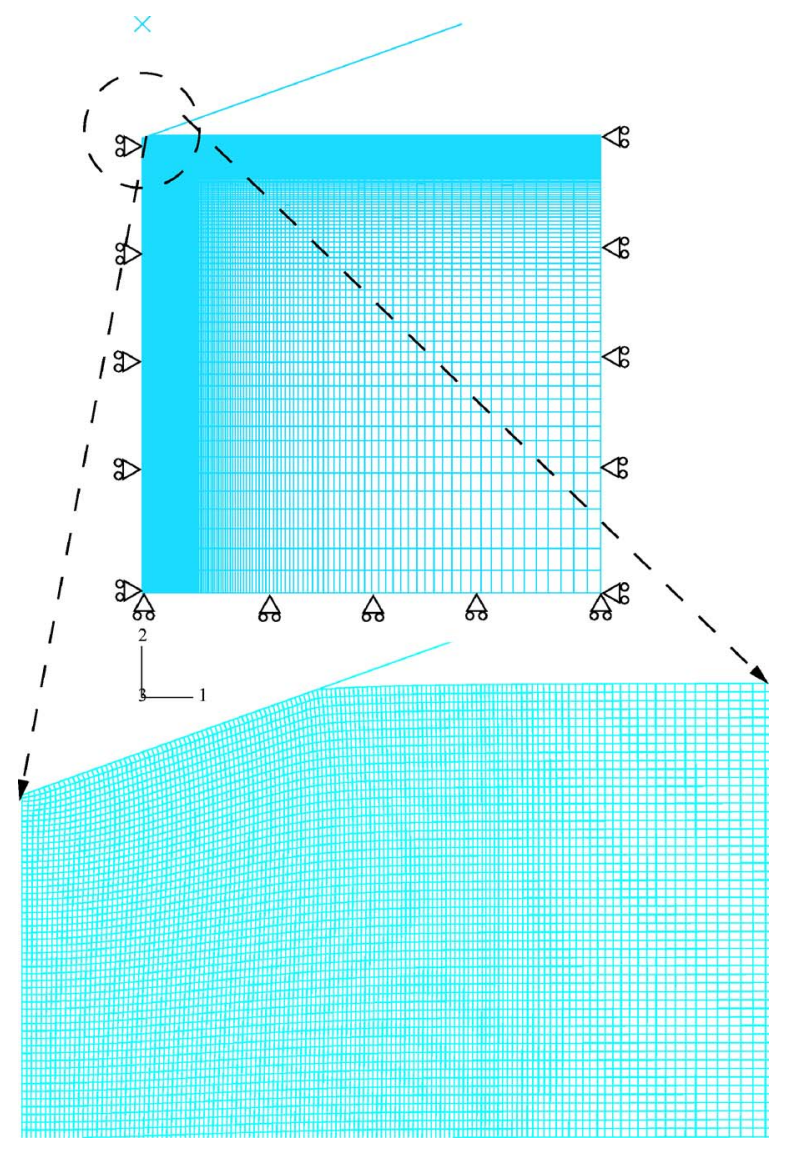

Fig. 2 Example of the axisymmetric mesh, including boundary conditions. The indenter is shown for maximum indentation depth.

modulus of the specimen. Altogether, the results shown in Figs. 3(a)-3(c) map the dimensionless space of indentation parameters, defining the problem.

\section{Reverse Analysis}

Based on the functions obtained from the FE simulations described above, the proposed method utilizes a reverse analysis to determine elastic-plastic properties and residual stresses from one indentation test. In this section, we will introduce the reverse analysis and finish with a comparison with experimental data to verify the proposed method.

4.1 Approximate Functional Form of $\Psi, \Pi$, and $\Omega$. Over the range of materials parameters studied in this paper $\left(E, \sigma_{y}, \sigma_{r e s}\right)$, the functional, normalized forms of the indentation parameters $\left(S, W_{u}\right.$, and $\left.W_{l}\right)$ were obtained from the FE simulations, presented in the previous section. These functions , $\Pi$, and $\Omega$ can be fitted into the following function:

$$
\begin{aligned}
\Phi= & a_{1}+a_{2} \tau+a_{3} \tau^{2}+a_{4} \sqrt{\tau}+a_{5} \sqrt[3]{\tau}+a_{6} \xi+a_{7} \xi \tau+a_{8} \xi \tau^{2}+a_{9} \xi \sqrt{\tau} \\
& +a_{10} \xi^{3} \bar{\tau}+a_{11} \xi^{2}+a_{12} \xi^{2} \tau+a_{13} \xi^{2} \tau^{2}+a_{14} \xi^{2} \sqrt{\tau}+a_{15} \xi^{2} \sqrt[3]{\tau} \\
& +a_{16} \xi^{3}+a_{17} \xi^{3} \tau+a_{18} \xi^{3} \tau^{2}+a_{19} \xi^{3} \sqrt{\tau} a_{20}+\xi^{3} \sqrt[3]{\tau}
\end{aligned}
$$

where the function $\Phi$ represents one of the three functions ( , $\Pi$, and $\Omega$ ), with $\tau \quad E / \sigma_{y}$ and $\xi \quad \sigma_{r e s} / \sigma_{y}$. The coefficients $a_{i}(i$ $=1-20)$ are listed in Table 1 . The fitted functions, $\Phi$, are superimposed with the functions surfaces obtained from the finite element analysis (,$\Pi$, and $\Omega$ ) and displayed in Figs. 3(a)-3(c). The errors between the fitted function compared to the data obtained from the finite element simulations are displayed in Figs. 3(d)-3(f). Even though some local regions exhibit significant errors (up to $20 \%$ for the normalized unloading work), the major part of the investigated parameter space exhibits less than $2 \%$ error. The fitting could be improved further by dividing the parameter space into several regions, for example develop one set of fitting functions for $E / \sigma_{Y}<500$ and another for $E / \sigma_{Y}>500$.

By minimizing the total square error of Eqs. (5)-(7), the three unknowns $\left(E, \sigma_{y}, \sigma_{\text {res }}\right)$ may be solved from reverse analysis through numerical iterations, illustrated with a flow chart in Fig. 4. The root-searching algorithm is based on the "golden section search" and "parabolic interpolation." Based on the results obtained from the last numerical step, the search boundary is updated automatically to give faster convergence. Thus, using this method, one starts with an estimated (guessed) value and lets the algorithm illustrated in Fig. 4 find the converged answer.

4.2 Numerical Examples of Reverse Analysis. In order to examine the (theoretical) accuracy of reverse analysis, several numerical experiments of indentation are performed, with $E / \sigma_{Y}$ and $\sigma_{\text {res }} / \sigma_{y}$ varying in a large range. ${ }^{3}$ The input material parameters used in the FE simulations are shown as black squares in Fig. 5. For each virtual experiment, the contact stiffness, the work of indentation, and the unload work are determined from the finite element analysis. These numbers are then fed into the reverse analysis (see the flow chart in Fig. 4) to predict $E / \sigma_{Y}$ and $\sigma_{r e s} / \sigma_{y}$. The results obtained from the reverse analysis are plotted as open circles in Fig. 5. Good agreements between the original input data and reverse analysis are found for all examined combinations of residual stress and material parameters investigated, in particular for values $E / \sigma_{Y}<600$. In all cases the normalized residual stress, $\sigma_{r e s} / \sigma_{y}$, is determined with relatively high accuracy. The error for materials with $E / \sigma_{Y}>600$ derives from the approximate fitting function and that all three functions $\quad, \Pi$, and $\Omega$ are relatively flat for $E / \sigma_{Y}>600$ (Fig. 3). Thus, the accuracy could be improved by adding more coefficients to Eq. (8) or dividing the input material space $\left(\sigma_{y} / E, \sigma_{r e s} / \sigma_{y}\right)$ used for functional fitting into different zones, so as to capture the functional form with higher precision. Alternatively, the current form can be used to establish an approximate value of $E / \sigma_{Y}$ followed by matching finite element simulations to achieve a more accurate value of $E / \sigma_{Y}$.

4.3 Sensitivity Analysis. Before using the method for real indentation tests, we will investigate the (theoretical) sensitivity of the method. In the reverse analysis discussed in Sec. 4.2 and illustrated in Fig. 5, the indentation parameters $\left(S, W_{l}\right.$, and $\left.W_{u}\right)$ were obtained from virtual experiments. In this case, the accuracy of the results depends on how well the fitting surfaces capture the true values, obtained from the extensive FEA presented in Sec. 3 . During physical experiments, there are potentially many sources of errors, resulting in measurement errors, particular for the contact stiffness. Thus, we will investigate the sensitivity of our method for errors in the measured parameters such as the indentation force and depth. To this end, we will conduct an error sensitivity analysis, where we perturb the values obtained from the virtual experiments and conduct a reverse analysis.

First, the contact stiffness, $S$, is given a small error while the other two parameters $\left(W_{l}\right.$ and $\left.W_{u}\right)$ are kept at the values obtained from the virtual experiments. Based on this perturbed set of parameters $\left\{\left.S\right|_{\text {error }},\left.W_{l}\right|_{\text {true }}\right.$, and $\left.\left.W_{u}\right|_{\text {true }}\right\}$, a reverse analysis is conducted to obtain the materials parameters. Three magnitudes of errors are investigated $\{ \pm 2 \%, \pm 5 \%, \pm 10 \%$,$\} and the results are$ summarized in Fig. 6(a). In Fig. 6(a), the original input and the reverse analysis from Fig. 5 are repeated. Superimposed on these are the six errors assumed on the contact stiffness. We note that the proposed method will strive to capture the circles, which are the point on the fitted surface (Figs. $3(a)-3(c)$ ). The method is quite insensitive for an input error for $E / \sigma_{Y}<400$, where the

${ }^{3}$ Note that none of these parametric combinations were used in generating Fig. 2 

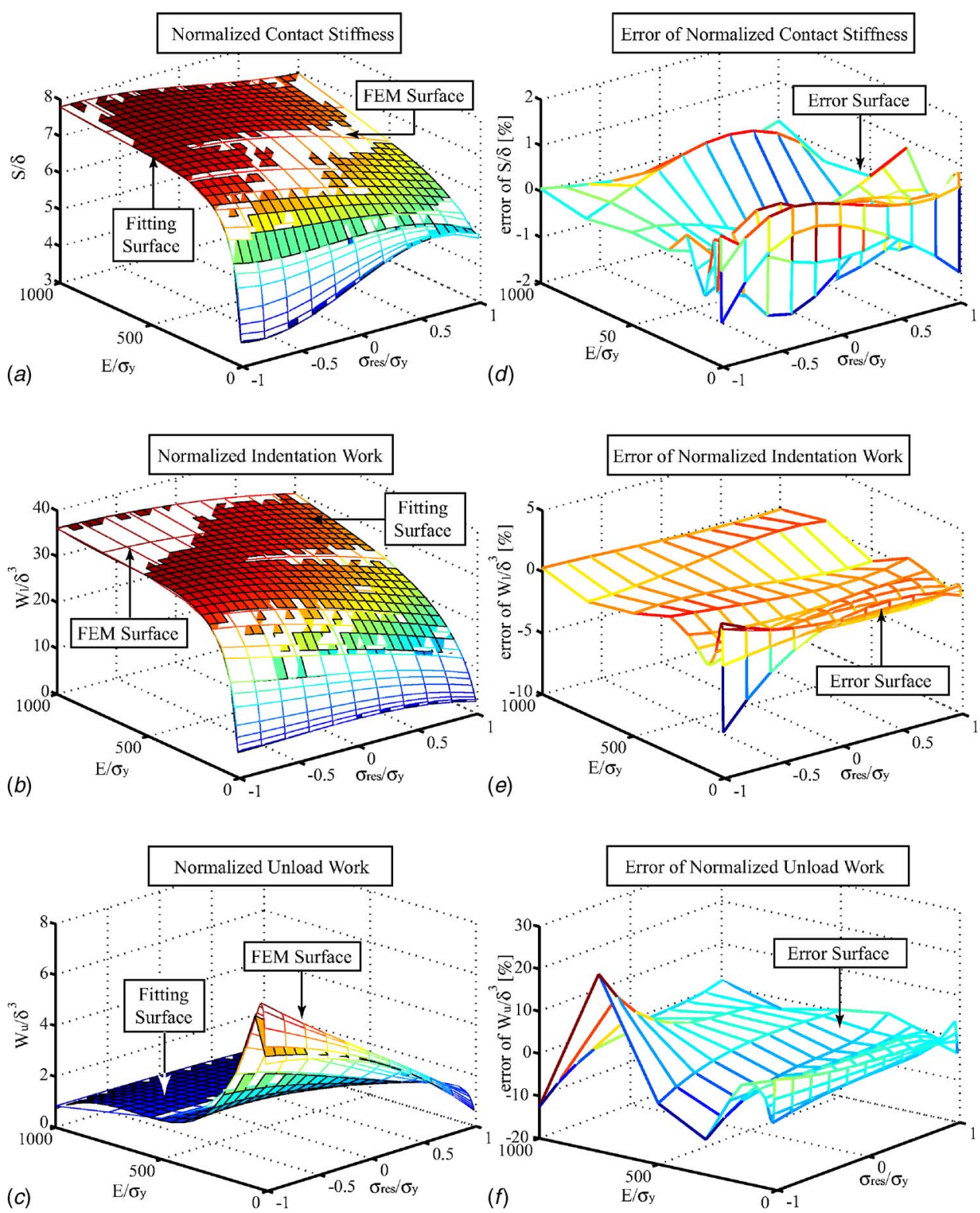

Fig. 3 The dimensionless functional forms based on the finite element simulations (a) contact stiffness, $\Psi,(b)$ work of indentation, $\Pi$, and (c) unload work, $\Omega$; the error between fitting functions and the functional forms (d) contact stiffness, $\Psi,(e)$ work of indentation, $\Pi$, and $(f)$ unload work, $\Omega$. The error is determined by (fitted-FEresults)/FEresults

obtained material properties for the various assumed errors overlap. For larger $E / \sigma_{Y}$, an increased sensitivity of input errors was observed, but the method tends to capture $\sigma_{r e s} / \sigma_{Y}$ well.

Next, the indentation work, $W_{l}$, is given a small error with the other two parameters $\left(S\right.$ and $\left.W_{u}\right)$ are kept at the values obtained from the virtual experiments. Similarly to the analysis described above, the perturbed set $\left\{S_{\text {true }}, W_{\text {lerror }}\right.$, and $\left.W_{u \text { true }}\right\}$ is used for the reverse analyses. In this case, the errors tend to be somewhat larger than when the contact stiffness was perturbed (Fig. 6(b) compared to Fig. 6(a)). We also note that in a practical experiment, the error of indentation work is likely to be smaller than that of contact stiffness, and a $10 \%$ perturbation of indentation work is unlikely to occur.

4.4 Comparison With Experiments. The error sensitivity analyses described in this subsection are based on idealized numerical simulations, including a sharp conical indenter on a semiinfinite linear-elastic, perfectly plastic material specimen. In real experiments, these idealizations will be compromised, with factors such as finite radius of the indentation tip, strain hardening, and finite size of the specimen. Therefore, care must be taken when using our proposed technique, to ensure the validity of the evaluations.

Experimental work is currently underway in our laboratory to verify the technique when a residual stress is present and will be reported shortly. Unfortunately, it is difficult to compare the present analyses with data available in the literature since the improved method requires the knowledge of force-displacement curves for both loading and unloading. Moreover, the proposed technique is specified for conical (Berkovich) indentation on linear-elastic, perfectly plastic bulk material (or thick coating) with equi-biaxial residual stress. ${ }^{4}$ In the literature, either the data

${ }^{4}$ Extending the proposed method to any other shape of the indenter is straightforward, but omitted for brevity. 
Table 1 Parameters of three fitting functional forms

\begin{tabular}{cccc}
\hline \hline Parameters & $\begin{array}{c}\text { Normalized } \\
\text { work of indentation } \\
\Pi\end{array}$ & $\begin{array}{c}\text { Normalized } \\
\text { contact stiffness }\end{array}$ & $\begin{array}{c}\text { Normalized } \\
\text { unload work } \\
\Omega\end{array}$ \\
\hline$a_{1}$ & -3.7438 & 1.6418 & -2.6038 \\
$a_{2}$ & -0.0771 & 0.0057 & 0.0418 \\
$a_{3}$ & $1.7110 \times 10^{-5}$ & $-1.4509 \times 10^{-6}$ & $-7.5492 \times 10^{-6}$ \\
$a_{4}$ & 2.9685 & -0.6657 & -3.4646 \\
$a_{5}$ & 0.4208 & 2.2608 & 7.8719 \\
$a_{6}$ & 8.9619 & 5.6491 & 7.6999 \\
$a_{7}$ & 0.0507 & -0.0012 & -0.0614 \\
$a_{8}$ & $-1.9059 \times 10^{-5}$ & $-1.3118 \times 10^{-6}$ & $1.1208 \times 10^{-5}$ \\
$a_{9}$ & 0.0963 & 0.9074 & 5.0438 \\
$a_{10}$ & -4.6897 & -3.2432 & -11.7033 \\
$a_{11}$ & -8.1284 & 0.6823 & -8.7370 \\
$a_{12}$ & 0.0270 & -0.0102 & 0.0437 \\
$a_{13}$ & $-2.0045 \times 10^{-6}$ & $3.1619 \times 10^{-6}$ & $-8.6205 \times 10^{-6}$ \\
$a_{14}$ & -2.7352 & 0.6009 & -3.5899 \\
$a_{15}$ & 6.8229 & -1.2696 & 8.7139 \\
$a_{16}$ & 5.9693 & -1.3395 & 1.1623 \\
$a_{17}$ & -0.0691 & -0.00743 & -0.0152 \\
$a_{18}$ & $1.6320 \times 10^{-5}$ & $2.3459 \times 10^{-6}$ & $4.0449 \times 10^{-6}$ \\
$a_{19}$ & 4.5106 & 0.2624 & 0.9069 \\
$a_{20}$ & -9.5823 & -0.1691 & -1.8675 \\
\hline \hline
\end{tabular}

are, for our purpose, incomplete, e.g., [7,9], or a spherical indenter is used [10]. In other experiments, the strain hardening/strain gradient effect is significant for metal specimens [11,12], involves non-negligible substrate effect for indentation on thin films [22],

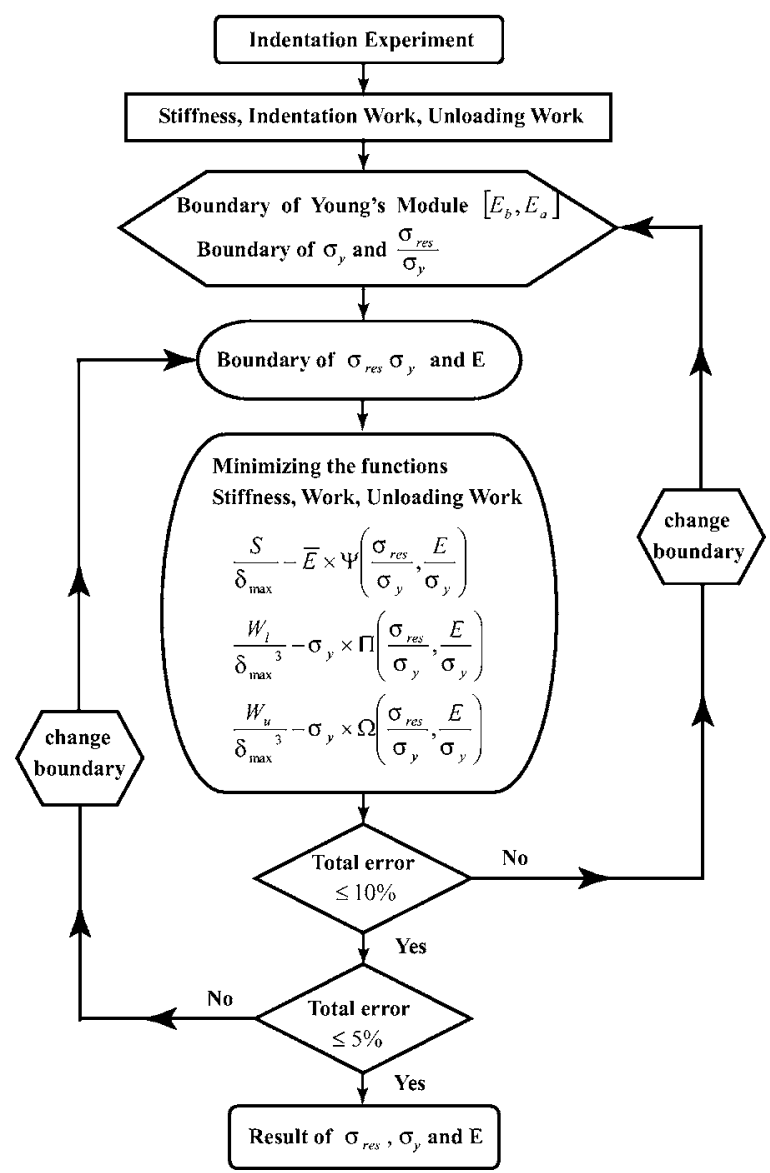

Fig. 4 Schematic of the process flow of reverse analysis

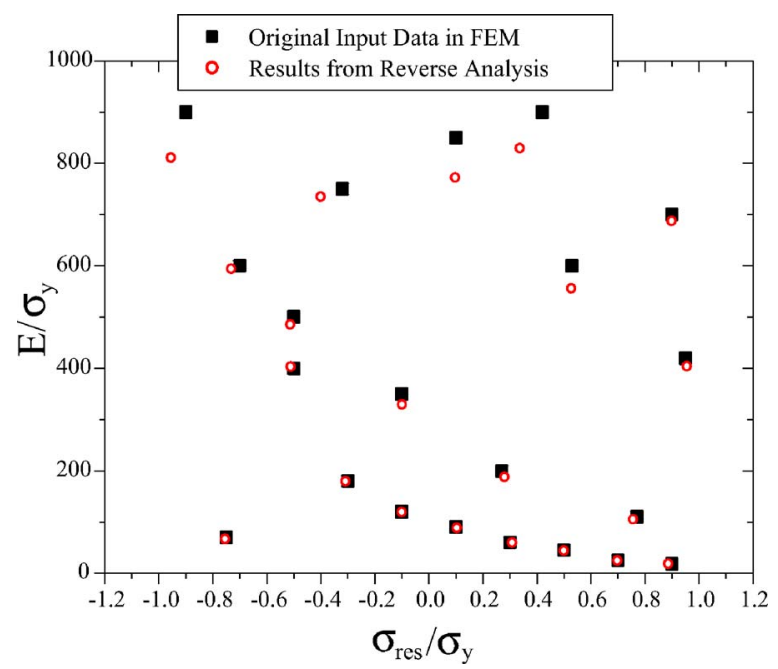

Fig. 5 Comparison between the material properties predicted from reverse analysis and the input parameters used in numerical indentation experiments for imaginary materials

or only uniaxial residual stress is present [14,23].

There are, however, limited complete data available in the literature pertaining to indentation testing of structures without residual stresses. Thus, we can use these tests to verify our proposed method for a subset of the parametric space the method is developed for. With these restrictions, we will use data from the instrumented indentation results reported in two papers for a range of materials. From Pelletier and Krier [24] we will use four materials: $99.7 \%$ nickel, $99.6 \%$ titanium, 316L stainless steel (70 wt.\% $\mathrm{Fe}, 18 \% \mathrm{Cr}, 11 \% \mathrm{Ni}, 1 \% \mathrm{Mo}$ ), and TAFe titanium alloy (4.5 wt.\% $\mathrm{Al}, 2.5 \% \mathrm{Fe}$, balance $\mathrm{Ti})$. In their experiments, a Berkovich diamond tip was used. Prior to indentation, the samples were well polished with a mean surface roughness of $30 \mathrm{~nm}$. From Schwarzer and Pharr [25], we will use the indentation curve from a Berkowich indentation with the maximum load of $50 \mathrm{mN}$ into fused silica. Young's modulus and the yield strength for each material are presented in Table 2. These properties are based on data the authors in the two papers use as reference data. The material data are published data from unrelated sources and not based on their indentation test.

Based on the force-displacement curves from the indentation testing presented in the relevant literature $[24,25]$, we used our methodology to determine Young's modulus and yield strength (Table 2). The errors between the "true" values and the values obtain from our algorithm are determined based on

$$
\text { Error }=\frac{\text { True-Calculated }}{\text { True }}
$$

where True corresponds to the reported values and Calculated corresponds to the value obtained by the method proposed in our paper, for Young's modulus and yield strength. These values are listed in Table 2.

The values obtained from the proposed method give small errors for nickel, iron, and fused silica: the error is less than $2 \%$ for the yield strength. However, the other three materials exhibit significant strain hardening (denoted by $E_{T}$ in Table 2), thus stretching the validity of our model.

In addition, we have compared our method with the commonly used evaluation technique presented in Eqs. (1) and (2). From Table 2 it is evident that the proposed method gives significantly better results than the traditional method for the majority of the cases. Thus, for materials with constitutive equations approaching linear-elastic, perfectly plastic response, our method gives better estimates than conventional methods that use the contact area as 

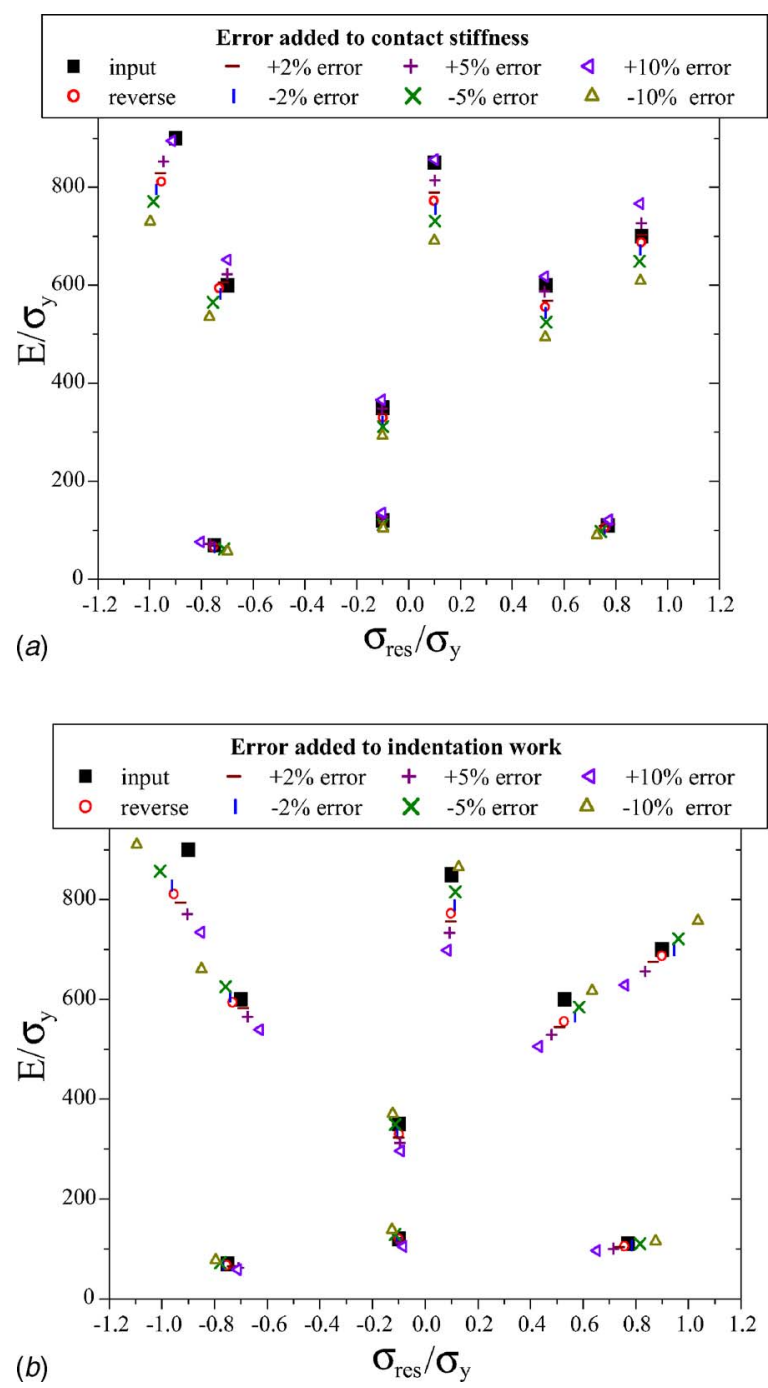

Fig. 6 Error sensitivity analysis with $2 \%, 5 \%$, and $10 \%$ error (a) in contact stiffness and (b) in indentation work: the reverse analysis is compared with input parameter from FE-simulations (squares) and the unperturbed analysis (circles) input. For materials with significant hardening behavior, the proposed method may lead to error and we are developing a new method to account for the work hardening effect, which has been submitted elsewhere [26]. One of the main error sources of the "Oliver-Pharr method" [1] is due to the error of contact area calculated from Eq. (2), which cannot account for the plastic pile-up. However, in our method, the measurement of contact area is not needed, which clearly demonstrates the advantage of the alternative technique presented in this paper.

Thus, we believe that the proposed method will be a useful method for real experimental investigations, since the proposed method will not rely on the contact area. However, in order to increase the accuracy of the results obtained, it is advisable to conducting verifying finite element simulations, in particular for materials with $E / \sigma_{Y}>600$.

\section{Conclusion}

An alternative method is proposed to measure the equi-biaxial residual stress and elastic-plastic properties of bulk materials or thick coatings by using conical microindentation. This method does not require measurement of a contact radius (contact area) and does not require a reference stress-free material. The dimensionless functional forms are established based on the contact stiffness, normalized indentation work, and work recovered during unloading. Using reverse analysis, we show that this method can be used to quickly and effectively determine the residual stress levels in a specimen.

To verify the model, published results for instrumented indentation tests are used. Complete results for materials subjecting to biaxial stresses could not be found. However, a limited set of complete data for stress free bulk materials was found and used for comparison. Using the data from the published forcedisplacement curves, we find excellent agreement from our proposed method to the published (reference) properties.

Thus, we believe that our proposed method of evaluating instrumented indentation testing of materials subjected to a biaxial residual stress will be a useful alternative method to evaluate an indentation test, without the requirement of determining the contact area during maximum indentation load.

\section{Acknowledgment}

The work of JY and AMK is supported by NSF Grant No. DMR-0346664 and ONR Grant No. N00014-04-1-0498. The

Table 2 Comparing results from the proposed method

\begin{tabular}{|c|c|c|c|c|c|c|c|c|c|}
\hline \multirow[b]{2}{*}{ Material } & \multicolumn{3}{|c|}{ Reference values } & \multicolumn{2}{|c|}{ Calculated values } & \multicolumn{2}{|c|}{ Error } & \multicolumn{2}{|c|}{ Traditional approach $^{\mathrm{b}}$} \\
\hline & $\begin{array}{l}\text { Young's } \\
\text { modulus } \\
(\mathrm{GPa})\end{array}$ & $\begin{array}{c}\text { Work-hardening } \\
\text { rate } \\
(\mathrm{GPa})^{\mathrm{a}}\end{array}$ & $\begin{array}{l}\text { Yield } \\
\text { strength } \\
\text { (MPa) }\end{array}$ & $\begin{array}{l}\text { Young's } \\
\text { modulus } \\
(\mathrm{GPa})\end{array}$ & $\begin{array}{l}\text { Yield } \\
\text { strength } \\
\text { (MPa) }\end{array}$ & $\begin{array}{l}\text { Young's } \\
\text { modulus } \\
(\%)\end{array}$ & $\begin{array}{l}\text { Yield } \\
\text { strength } \\
(\%)\end{array}$ & $\begin{array}{l}\text { Young's } \\
\text { modulus } \\
(\mathrm{GPa})\end{array}$ & $\begin{array}{c}\text { Error } \\
(\%)\end{array}$ \\
\hline $\begin{array}{l}\text { Bulk Ni } \\
{[20]}\end{array}$ & 240 & 19 & 530 & 225.4 & 536.7 & 6.25 & 1.32 & 299.0 & 24.4 \\
\hline $\begin{array}{l}\text { Bulk Fe } \\
{[20]}\end{array}$ & 215 & 26 & 200 & 197.65 & 202.7 & 7.90 & 1.50 & 272.0 & 26.6 \\
\hline $\begin{array}{l}\text { Bulk Ti } \\
{[20]}\end{array}$ & 130 & 36 & 600 & 113.2 & 666.15 & 13.0 & 11.0 & 149.0 & 14.6 \\
\hline $\begin{array}{l}\text { Bulk TAFe } \\
{[20]}\end{array}$ & 110 & 44 & 795 & 124.7 & 754.8 & 13.6 & 5.03 & 156 & 41.6 \\
\hline $\begin{array}{l}\text { Bulk A316L } \\
{[20]}\end{array}$ & 210 & 31.5 & 195 & 173.53 & 204.13 & 17.6 & 4.62 & 203 & 3.45 \\
\hline $\begin{array}{l}\text { Fused silica } \\
\text { [21] }\end{array}$ & 72 & $\cdots$ & 7050 & 69.3 & 6930 & 3.75 & 1.70 & 85.7 & 19.0 \\
\hline
\end{tabular}

${ }^{\mathrm{a}}$ In [20] the work hardening rate, $E_{T}$, was used as a measure of work hardening, defined according to the following: The stress-strain curve is defined by a continuous curve, divided into two linear regions and connected to a stress value corresponding to the yield strength. For lower stresses, the curve describes the elastic behavior with the slope being Young's modulus, E. For higher stresses, above the yield strength, the line describes the nonelastic response. The slope of this curve is referred to as work-hardening rate the value of which is given in the table.

Using the classical method according to Eqs. (1) and (2) 
work of $\mathrm{XC}$ is supported in part by NSF Grant No. CMS0407743

\section{References}

[1] Oliver, W. C., and Pharr, G. M., 1992, "An Improved Technique for Determining Hardness and Elastic-Modulus Using Load and Displacement Sensing Indentation Experiments," J. Mater. Res., 7, pp. 1564-1583.

[2] Johnson, K. L., 1985, Contact Mechanics, Cambridge U.P., Cambridge.

[3] Hay, J. C., Bolshakov, A., and Pharr, G. M., 1999, “A Critical Examination of the Fundamental Relations Used in the Analysis of Nanoindentation Data," J. Mater. Res., 14, pp. 2296-2305.

[4] Chen, X., Yan, J., and Karlsson, A. M., 2006, "On the Determination of Residual Stress and Mechanical Properties by Indentation,” Mater. Sci. Eng., A, 416, pp. 139-149.

[5] Pharr, G. M., 1998, "Measurement of Mechanical Properties by Ultra-low Load Indentation," Mater. Sci. Eng., A, 253, pp. 151-159.

[6] Xu, Z.-H., and Li, X., 2005, "Influence of Equi-biaxial Residual Stress on Unloading Behaviour of Nanoindentation," Acta Mater., 53, pp. 1913-1919.

[7] Tsui, T. Y., Oliver, W. C., and Pharr, G. M., 1996, "Influences of Stress on the Measurement of Mechanical Properties Using Nanoindentation. 1. Experimental Studies in an Aluminum Alloy," J. Mater. Res., 11, pp. 752-759.

[8] Bolshakov, A., Oliver, W. C., and Pharr, G. M., 1996, "Influences of Stress on the Measurment of Mechanical Properties Using Nanoindentation. 2. Finite Element Simulations,” J. Mater. Res., 11, pp. 760-768.

[9] Suresh, S., and Giannakopoulos, A. E., 1998, "A New Method for Estimating Residual Stresses by Instrumented Sharp Indenter," Acta Mater., 46, pp. 5755-5767.

[10] Swadener, J. G., Taljat, B., and Pharr, G. M., 2001, "Measurement of Residual Stress by Load and Depth Sensing Indentation With Spherical Indenters," J. Mater. Res., 16, pp. 2091-2102.

[11] Lee, Y. H., and Kwon, D., 2003, "Measurment of Residual-Stress by Nanoindentation on Elastically Strained (100) W," Scr. Mater., 49, pp. 459-465.

[12] Lee, Y. H., and Kwon, D., 2004, "Estimation of Biaxial Surface Stress by Instrumented Indentation With Sharp Indenters," Acta Mater., 53, pp. 15551563.
[13] Carlsson, S., and Larsson, P. L., 2001, "On the Determination of Residual Stress and Strain Fields by Sharp Indentation Testing. Part I: Theoretical and Numerical Analysis," Acta Mater., 49, pp. 2179-2191.

[14] Carlsson, S., and Larsson, P. L., 2001, "On the Determination of Residual Stress and Strain Fields by Sharp Indentation Testing. Part II: Experimental Investigation," Acta Mater., 49, pp. 2193-2203.

[15] Chen, X., and Vlassak, J. J., 2001, "Numerical Study on the Measurement of Thin Film Mechanical Properties by Means of Nanoindentation," J. Mater. Res., 16, pp. 2974-2982.

[16] Mesarovic, S. D., and Fleck, N. A., 1999, "Spherical Indentation of ElasticPlastic Solids," Proc. R. Soc. London, Ser. A, 455, pp. 2707-2728

[17] ABAQUS, 2004, "ABAQUS 6.5," ABAQUS Inc., Pawtucket, RI.

[18] Bowden, F. P., and Tabor, D., 1950, The Friction and Lubrications of Solids, Oxford U.P., Oxford.

[19] Cheng, Y., and Cheng, C., 2004, "Scaling, Dimensional Analysis, and Indentation Measurements," Mater. Sci. Eng., R., 44, pp. 91-149.

[20] Bucaille, J., Stauss, S., Felder, E., and Michler, J., 2003, "Determination of Plastic Properties of Metals by Instrumented Indentation Using Different Sharp Indenters," Acta Mater., 51, pp. 1663-1678.

[21] Wang, L., and Rokhlin, S., 2005, "Universal Scaling Functions for Continuous Stiffness Nanoindentation With Sharp Indenters," Int. J. Solids Struct., 42, pp. 3807-3832.

[22] Wei, G., Bhushan, B., and Jacobs, S., 2004, "Nanomechanical Characterization of Multilayered Thin Film Structures for Digital Micromirror Devices," Ultramicroscopy, 100, pp. 375-389.

[23] Zagrebelny, A. V., and Carter, C. B., 1997, "Indentation of Strained SilicateGlass Films on Alumina Substrates," Scr. Mater., 37, pp. 1869-1875.

[24] Pelletier, H., Krier, J., Cornet, A., and Mille, P., 2000, "Limits of Using Bilinear Stress-Strain Curve for Finite Element Modeling of Nanoindentation Response on Bulk Materials," Thin Solid Films, 379, pp. 147-155.

[25] Schwarzer, N., and Pharr, G., 2004, "On the Evaluation of Stresses During Nanoindentation With Sharp Indenters," Thin Solid Films, 469-70, pp. 194200.

[26] Yan, J., Karlsson, A. M., and Chen, X., 2006, "Determining Plastic Properties of a Bulk Material With Residual Stress by Using Conical Indentation," Int. J. Solids Struct., in press. 